\title{
An improved allele-specific PCR primer design method for SNP marker analysis and its application
}

Jing Liư ${ }^{1 \dagger}$, Shunmou Huang ${ }^{1 \dagger}$, Meiyu Sun ${ }^{1}$, Shengyi Liu', Yumei Liu², Wanxing Wang ${ }^{2}$, Xiurong Zhang ${ }^{1}$, Hanzhong Wang ${ }^{1}$ and Wei Hua ${ }^{1 *}$

\begin{abstract}
Background: Although Single Nucleotide Polymorphism (SNP) marker is an invaluable tool for positional cloning, association study and evolutionary analysis, low SNP detection efficiency by Allele-Specific PCR (AS-PCR) still restricts its application as molecular marker like other markers such as Simple Sequence Repeat (SSR). To overcome this problem, primers with a single nucleotide artificial mismatch introduced within the three bases closest to the 3'end (SNP site) have been used in AS-PCR. However, for one SNP site, nine possible mismatches can be generated among the three bases and how to select the right one to increase primer specificity is still a challenge.

Results: In this study, different from the previous reports which used a limited quantity of primers randomly (several or dozen pairs), we systematically investigated the effects of mismatch base pairs, mismatch sites and SNP types on primer specificity with 2071 primer pairs, which were designed based on SNPs from Brassica oleracea 01-88 and 02-12. According to the statistical results, we (1) found that the primers designed with SNP (A/T), in which the mismatch (CA) in the $3^{\text {rd }}$ nucleotide from the $3^{\prime}$ end, had the highest allele-specificity (81.9\%). This information could be used when designing primers from a large quantity of SNP sites; (2) performed the primer design principle which forms the one and only best primer for every SNP type. This is never reported in previous studies. Additionally, we further identified its availability in rapeseed (Brassica napus L.) and sesame (Sesamum indicum). High polymorphism percent (75\%) of the designed primers indicated it is a general method and can be applied in other species.
\end{abstract}

Conclusion: The method provided in this study can generate primers more effectively for every SNP site compared to other AS-PCR primer design methods. The high allele-specific efficiency of the SNP primer allows the feasibility for low- to moderate- throughput SNP analyses and is much suitable for gene mapping, map-based cloning, and marker-assisted selection in crops.

Keywords: SNP, AS-PCR, Mismatch, Polymorphism, Destabilization

\section{Introduction}

Single Nucleotide Polymorphisms (SNPs) are single base differences between DNA of different individuals. Once discovered, SNPs can be converted into genetic markers that can be assayed $[1,2]$. As the most abundant and stabile form of genetic variation in most organism

\footnotetext{
* Correspondence: huawei@oilcrops.cn

${ }^{\dagger}$ Equal contributors

'Key Laboratory of Biology and Genetic Improvement of Oil Crops, Ministry of Agriculture, Oil Crops Research Institute of the Chinese Academy of

Agricultural Sciences, Wuhan 430062, People's Republic of China

Full list of author information is available at the end of the article
}

genomes, SNPs are more suitable for genotyping markers compared to the conventional markers such as RFLP (Restriction fragment length polymorphism), AFLP (Amplified fragment length polymorphism) and SSR (Simple Sequence Repeat). With the development of bio-technology, SNPs are becoming favored genetic markers that are used in marker-assisted breeding [3], mapbased cloning [4], study of evolutionary conservations between different species [5,6], and the detection of riskassociated alleles linked to human diseases [7].

\section{Biomed Central}

(C) 2012 Liu et al.; licensee BioMed Central Ltd. This is an Open Access article distributed under the terms of the Creative Commons Attribution License (http://creativecommons.org/licenses/by/2.0), which permits unrestricted use, distribution, and reproduction in any medium, provided the original work is properly cited. 
Recently, massive parallel sequencing platforms such as GSFLX (Roche), Solexa (Illumina) and SOLID (Applied Bios stems) have significantly reduced the cost of high throughout sequencing [8]. A large number of genomes and transcriptomes have been rapidly sequenced using these new platforms to identify novel SNPs in maize [9], rapeseed [10] and human [11] etc. A large variety of techniques for high-throughput SNP genotyping have also been developed using Taqman [12], Amplifluor [13], genome re-sequencing [14,15], and SNP arrays $[16,17]$. These techniques are expensive and require specialized equipments, which cost more standard primers and are not practical for assaying low- to moderate-throughput SNPs. Hence, there is a need for simple and accurate genotyping assays that can be implemented in laboratories lacking access to sophisticated equipment.

Traditional SNP genotyping methods such as CAPs (The Cleaved Amplified Polymorphic Sequence), dCAPs (derived CAPS), and AS-PCR (Allele-specific PCR) are widely used for low-throughput applications in plant research. In application, CAPS and dCAPS are restricted by end nuclease sites that could be inefficient and not cost-effective [18-20]. AS-PCR is based on the extension of primer only when its 3'end is a perfectly complemented to the template [21]. In principle, SNPs can be detected using allele-specific PCR primers based on the 3 ' terminal nucleotide of a primer that corresponds to a specific SNP site. However, reliable discrimination between the alleles is not sufficient to achieve using this described method. To overcome this problem, allelespecific primers with an additional base pair change within the three bases closest to the SNP site between alleles have been used [21,22]. Each specific SNP site in an allele can generate at least 18 possible primers with one mismatch base [23]. The SNAPER program generates a list of up to 16 possible primers per SNP site for each allele [23]. Therefore, choosing additional mismatches to increase primer specificity has been a challenge for AS-PCR [23]. Some studies have proposed criteria for designing AS-PCR primers. Hayashi et al (2004) proposed that base pair mismatches created through T-G or C-A transversions at third base from 3' end could increase the allele-specificity [24]. Hirotsu et al (2010) identified A-T transversion and A-G transition were useful base pair mismatches for improvement of allele-specific amplification [25]. The WASP tool could also be used to introduce mismatches at the penultimate $\left(2^{\text {nd }}\right.$ to the terminal) base of the primer $[26,27]$. However, most studies used only a limited quantity of primers, which might have some influences on efficiency of SNP primer specificity.

In this study, over 2000 primer pairs, which were designed based on SNPs between B. oleracea lines 01-88 and 02-12, were used to analyze the effects of different SNP types, mismatch bases and sites within the three bases closest to the 3'end on primer specificity. Based on these results, we advanced the SNP primer design principle. Compared to traditional SNP genotyping methods, our method could provide a cost-effective alternative for high efficient specific primers and would greatly facilitate plant research.

\section{Results}

\section{SNP analysis of B.oleracea 01-88 and 02-12 genome} sequences

The assembly of genome sequences of B.oleracea line 02-12 has been accomplished (unpublished). To identify SNPs between B. oleracea lines 01-88 and 02-12, genome DNA of line 01-88 was re-sequenced and a total of 119 million reads were obtained. To get high-quality SNPs, the sequence data was subjected to stringent filtering: The reads from line 01-88 were compared to the sequences of line 02-12 using BLASTN. Sites containing

Table 1 Putative SNPs identified between B. oleracea genomes of 01-88 and 02-12

\begin{tabular}{|c|c|c|c|}
\hline SNP $(01-88 / 02-12)$ & No. of every SNP type & Caused by & Total No. of transition and transversion \\
\hline A/G & 201247 & transition & 806387 \\
\hline $\mathrm{C} / \mathrm{T}$ & 202316 & & \\
\hline$G / A$ & 202801 & transition & \\
\hline $\mathrm{T} / \mathrm{C}$ & 200023 & & \\
\hline $\mathrm{A} / \mathrm{C}$ & 79813 & transversion & 615726 \\
\hline $\mathrm{G} / \mathrm{T}$ & 79800 & & \\
\hline $\mathrm{C} / \mathrm{A}$ & 80029 & transversion & \\
\hline T/G & 79782 & & \\
\hline $\bar{A} / \mathrm{T}$ & 99848 & transversion & \\
\hline T/A & 99743 & & \\
\hline $\mathrm{G} / \mathrm{C}$ & 48372 & transversion & \\
\hline $\mathrm{C} / \mathrm{G}$ & 48339 & & \\
\hline
\end{tabular}


tri-allelic or high degree of polymorphism were omitted. The sequences containing over 8 reads mapped to unique sites in the genome sequence were extracted from line 01-88. Pair wise alignment was used to evaluate the SNPs between genome sequences of 01-88 and 02-12. The alignment result revealed a total of 1,422,113 SNPs existed between B. oleracea lines 01-88 and 02-12 (an average of one SNP in every $360 \mathrm{bp}$ fragment) (Table 1). Analysis to the SNPs showed over half (56.7\%, 806387/1422113) of the nucleotide changes were transitions (A-G or C-T). Transversions (A-T, A-C, C-G and G-T) accounted for $43.3 \%(615726 / 1422113)$ of the detected SNPs (Table 1).

To further identify the putative SNPs and estimate the proportion of false positives, 96 SNP sites derived from only 8-read sequences were chosen randomly. Primers were designed according to the genome sequences near these SNP sites and all amplicons could generate about $500 \mathrm{bp}$ fragments in which containing the corresponding SNPs. Sanger sequencing results showed 93 SNPs were identical with the putative SNPs and 3 SNPs were unpredicted or undetectable. This indicated a very high proportion of SNPs really existed between lines 01-88 and $02-12$.

\section{Design and efficiency detection of SNP primers}

Primer design strategy for allele-specific PCR was illustrated in Figure 1. Primer P1, which was designed based on 02-12 genome sequence, formed a mismatch (TG) in 3'end with the DNA sequence of 01-88. While in most cases, it still could amplify the band with 01-88 efficiently. After introducing another mismatch (GA) in the $2^{\text {nd }}$ site closest to the 3 'end in primer P2, it could only amplify the band with 02-12 (Figure 1a). Based on the method described by Cha et al (1992), mismatch sites and mismatch bases closest to the SNP site were randomly chosen [21]. Therefore, nine possible mismatches could be generated among the three bases closest to 3 'end of primer (Figure 1b).

In this study, 1686 B. oleracea SNPs including 12 kinds of SNPs were chosen for primer design (Additional file 1). Among them, some of those SNPs could form the same 3'end mismatches in 01-88 with allele-specific primers. Therefore, all SNPs could be classified into eight kinds including $\mathrm{A} / \mathrm{G}$ and $\mathrm{C} / \mathrm{T}, \mathrm{T} / \mathrm{C}$ and $\mathrm{G} / \mathrm{A}, \mathrm{A} / \mathrm{C}$ and $\mathrm{G} / \mathrm{T}, \mathrm{T} / \mathrm{G}$ and $\mathrm{C} / \mathrm{A}, \mathrm{A} / \mathrm{T}, \mathrm{T} / \mathrm{A}, \mathrm{C} / \mathrm{G}, \mathrm{G} / \mathrm{C}$ (Table 2) based on mismatch types (for example, for SNPs A/G and C/T, the 3' end mismatch of the allele-specific primer designed for 02-12 is TG in 01-88). Moreover, for convenience of data analysis, we further compressed into four SNP types including A/G (T/C), A/C (T/G), A/T (T/A) and G/C (C/G) based on their destabilization effects in mismatch base pairs (Table 2) [28,29].

In this study, all the primers were used to amplify the genome DNA of lines 01-88 and 02-12, and PCR products were detected on $2.5 \%$ agarose gel by electrophoresis. The

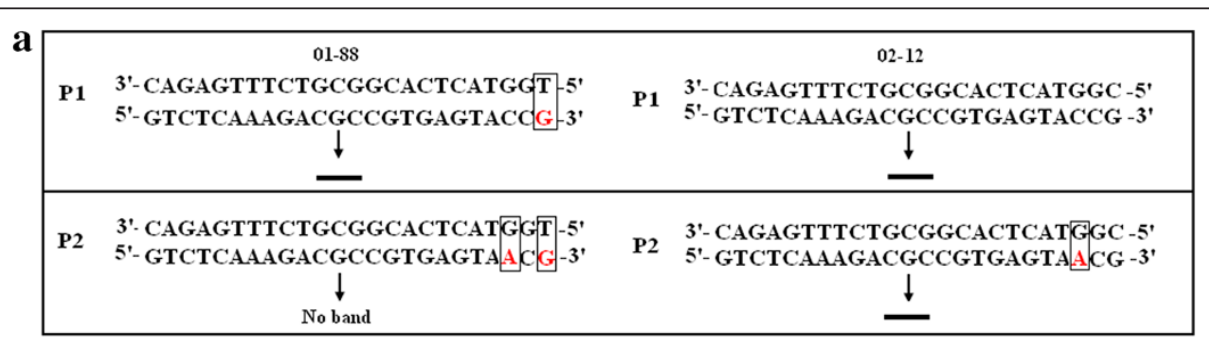

b mismatch site within three bases

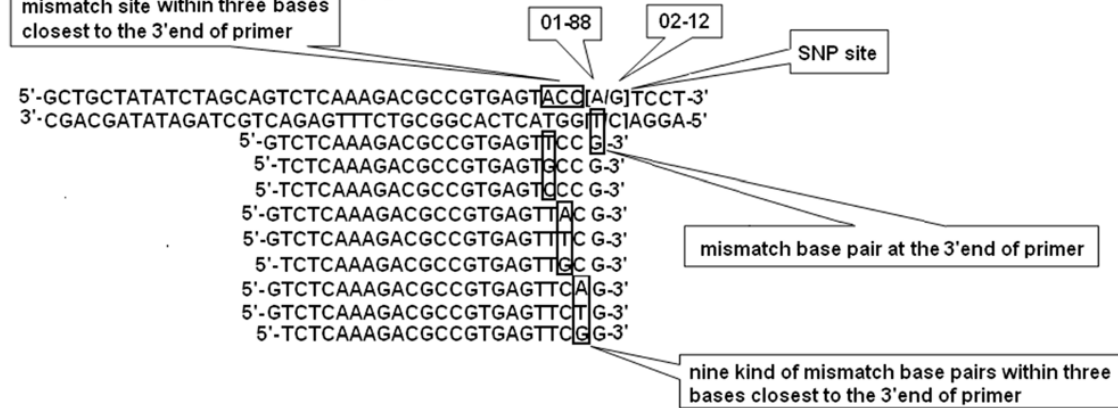

Figure 1 Schematic representation of the AS-PCR primer design. a, Primer P1 forms a perfect match with allele from 02-12, but a mismatch base pair at the 3'end with the DNA sequence of allele from 01-88. It could amplify the band in both of two lines 01-88 and 02-12. Primer P2 forms two mismatch base pairs with allele from $01-88$ at the $3^{\prime}$ end and in the $3^{\text {rd }}$ nucleotide from the $3^{\prime}$ end, while a mismatch base pair in the $3^{\text {rd }}$ nucleotide with allele from 02-12. It amplified the band only in 02-12. b. Schematic representation of different mismatches during the SNP primer design. 
Table 2 Destabilization strength of eight combinations of mismatch nucleotide pairing

\begin{tabular}{|c|c|c|}
\hline $\begin{array}{l}\text { SNP types } \\
(01-88 / 02-12)\end{array}$ & $\begin{array}{c}\text { mismatch } \\
\text { types at } \\
3^{\prime} \text { end of } \\
\text { primer in } \\
01-88\end{array}$ & $\begin{array}{l}\text { Destabilization } \\
\text { strength of } \\
\text { mismatch type }\end{array}$ \\
\hline$A / G$ & TG & Strong \\
\hline $\mathrm{C} / \mathrm{T}$ & GT & \\
\hline $\mathrm{G} / \mathrm{A}$ & $A C$ & \\
\hline$T / C$ & CA & \\
\hline$A / C$ & TC & Weak \\
\hline $\mathrm{G} / \mathrm{T}$ & CT & \\
\hline $\mathrm{C} / \mathrm{A}$ & AG & \\
\hline$T / G$ & GA & \\
\hline$A / T$ & $\mathrm{TT}$ & Strong and Medium \\
\hline$T / A$ & AA & \\
\hline $\mathrm{G} / \mathrm{C}$ & CC & Strong and Medium \\
\hline $\mathrm{C} / \mathrm{G}$ & GG & \\
\hline
\end{tabular}

results showed that among the 1686 primer pairs, 490 pairs (29.1\%) displayed polymorphism (Table 3 ). When classified by SNP types, the percents of polymorphism of A/G (C/T), A/C (G/T), A/T (T/A), C/G (G/C) were $26.4 \%$ (111/420), $31.3 \%$ (131/418), $34 \%(148 / 435)$ and $37.5 \%$ (155/413) respectively. When classified by mismatch sites, polymorphism percents of the $2^{\text {nd }}, 3^{\text {rd }}$ and $4^{\text {th }}$ base location closest to the SNP sites were 25.1\% (139/554), 32.9\% $(189 / 575)$ and $29.1 \%(162 / 557)$, respectively. If SNP type and mismatch site in primers were both considered, the polymorphism percent of SNP A/T (T/A) which had mismatches in the $3^{\text {rd }}$ site was $45.9 \%(72 / 157)$, followed by C/ $\mathrm{G}(\mathrm{G} / \mathrm{C})$ in the $2^{\text {nd }}$ site with $43.3 \%(58 / 134)$ and $3^{\text {rd }}$ site with $39.4 \%$ (56/142). The polymorphism percent of SNP $\mathrm{A} / \mathrm{G}(\mathrm{C} / \mathrm{T})$ was $26.4 \%(111 / 420)$, which also has the lowest polymorphisms in all mismatch sites.

Moreover, the polymorphism percents of SNP primers classified by the types of mismatch bases in the $2^{\text {nd }}, 3^{\text {rd }}$ and $4^{\text {th }}$ mismatch sites were analyzed (Table 4 ). Results showed that polymorphism percents of 8 mismatch types were closely equivalent in the $2^{\text {nd }}$ sites $(22.5 \%$ $26.4 \%$ ). High polymorphism percents appeared in two mismatch base pairs in the $3^{\text {rd }}$ base location (CA, 46.8\%, 37/79; TG, 38.6\%, 27/70). At the $4^{\text {th }}$ base away from the SNP site, the polymorphism percents of primers with mismatch base pairs GA, TC, TT and CC were over 30\% (31.7\%-33.3\%).

Based on the results in Table 3 and Table 4, we inferred that primers, which had CA mismatch in the $3^{\text {rd }}$ base closest to SNP site A/T (T/A), had the highest polymorphism between lines $01-88$ and 02-12. To identify this conjecture, 385 primers designed from SNP A/T (T/A), which contained $\mathrm{G}$ or $\mathrm{T}$ base in the $3^{\text {rd }}$ nucleotide, were further chosen for polymorphism analysis (Additional file 2). The detection results showed that 295 SNP primers were polymorphic $(295 / 385,76.6 \%)$ between lines 01-88 and 02-12. When these primers were classified based on the SNPs (A/T or T/A) in 3'end, the specificity percent of SNP (A/T) $(158 / 193,81.9 \%)$ was higher than that of SNP (T/A) (137/192, 71.4\%) (Figure 2a and Figure 2b).

\section{Application of the design method}

Besides the highest polymorphic primers mentioned above, the high polymorphic primers could be found in every SNP type based on our results. The principle of primer design is described as followed: firstly, for every kind of SNP, the mismatch site (the $2^{\text {nd }}, 3^{\text {rd }}$ and $4^{\text {th }}$ site closest to the SNP site) is chosen according to the result of polymorphism percent in Table 3. For the primers of three SNP types A/T (T/A), A/C (G/T) and A/G (C/T), the highest polymorphism percents are $45.9 \%, 37.4 \%$ and $30.7 \%$, respectively and the mismatches in primers are all located in the $3^{\text {rd }}$ site. While for the SNP type C/G (G/C), the mismatches in the $2^{\text {nd }}$ site closest to 3'end of primers show highest polymorphism percent (43.3\%). For the base

Table 3 Effect of mismatch sites and SNP types on the specificity of allele-specific PCR

\begin{tabular}{|c|c|c|c|c|c|}
\hline \multirow{2}{*}{$\begin{array}{l}\text { Mismatch } \\
\text { sites closest } \\
\text { to the } 3 \text { 'end } \\
\text { of primers }\end{array}$} & \multirow{2}{*}{$\begin{array}{l}\text { Polymorphism } \\
\text { percent of SNP } \\
\text { primers }\end{array}$} & \multicolumn{4}{|c|}{ Polymorphism percent of primers for every SNP type } \\
\hline & & $A / G, C / T$ & $\mathrm{~A} / \mathrm{C}, \mathrm{G} / \mathrm{T}$ & $\mathrm{A} / \mathrm{T}, \mathrm{T} / \mathrm{A}$ & $\mathrm{C} / \mathrm{G}, \mathrm{G} / \mathrm{C}$ \\
\hline \multirow[t]{2}{*}{ The $2^{\text {nd }}$ base } & $25.1 \%$ & $23.0 \%$ & $26.8 \%$ & $26.6 \%$ & $43.3 \%$ \\
\hline & $(139 / 554)$ & $(32 / 139)$ & $(37 / 138)$ & $(38 / 143)$ & $(58 / 134)$ \\
\hline \multirow[t]{2}{*}{ The $3^{\text {rd }}$ base } & $32.9 \%$ & $30.7 \%$ & $37.4 \%$ & $45.9 \%$ & $39.4 \%$ \\
\hline & $(189 / 575)$ & $(42 / 137)$ & $(52 / 139)$ & $(72 / 157)$ & $(56 / 142)$ \\
\hline \multirow[t]{2}{*}{ The $4^{\text {th }}$ base } & $29.1 \%$ & $25.7 \%$ & $29.8 \%$ & $28.1 \%$ & $29.9 \%$ \\
\hline & $(162 / 557)$ & $(37 / 144)$ & $(42 / 141)$ & $(38 / 135)$ & $(41 / 137)$ \\
\hline \multirow[t]{2}{*}{ Total number } & $29.1 \%$ & $26.4 \%$ & $31.3 \%$ & $34 \%$ & $37.5 \%$ \\
\hline & $(490 / 1686)$ & $(111 / 420)$ & $(131 / 418)$ & $(148 / 435)$ & $(155 / 413)$ \\
\hline
\end{tabular}


Table 4 Effect of artificial base mismatches in three mismatch sites on the specificity of allele-specific PCR

\begin{tabular}{|c|c|c|c|c|c|c|c|c|}
\hline $\begin{array}{l}\text { Mismatch sites } \\
\text { closest to the } 3^{\prime} \text { end } \\
\text { of primers }\end{array}$ & $\begin{array}{l}\text { Mismatch } \\
\text { types }\end{array}$ & $\begin{array}{l}\text { Polymorphism } \\
\text { percent of } \\
\text { primers }\end{array}$ & $\begin{array}{l}\text { Mismatch } \\
\text { types }\end{array}$ & $\begin{array}{l}\text { Polymorphism } \\
\text { percent of } \\
\text { primers }\end{array}$ & $\begin{array}{l}\text { Mismatch } \\
\text { types }\end{array}$ & $\begin{array}{l}\text { Polymorphism } \\
\text { percent of } \\
\text { primers }\end{array}$ & $\begin{array}{l}\text { Mismatch } \\
\text { types }\end{array}$ & $\begin{array}{l}\text { Polymorphism } \\
\text { percent of } \\
\text { primers }\end{array}$ \\
\hline \multirow[t]{4}{*}{ The $2^{\text {nd }}$ base } & TC & $26.2 \%$ & GA & $22.5 \%$ & CA & $25.7 \%$ & AA & $23.8 \%$ \\
\hline & & $(17 / 65)$ & & $(16 / 71)$ & & $(19 / 74)$ & & $(15 / 63)$ \\
\hline & TG & $26.4 \%$ & GG & $24.2 \%$ & CC & $26.1 \%$ & $T T$ & $25.6 \%$ \\
\hline & & $(19 / 72)$ & & $(15 / 62)$ & & $(18 / 69)$ & & $(20 / 78)$ \\
\hline \multirow[t]{4}{*}{ The $3^{\text {rd }}$ base } & TC & $32.1 \%$ & GA & $28.3 \%$ & CA & $46.8 \%$ & AA & $30.6 \%$ \\
\hline & & $(26 / 81)$ & & $(17 / 60)$ & & $(37 / 79)$ & & $(26 / 85)$ \\
\hline & TG & $38.6 \%$ & GG & $30.4 \%$ & CC & $23.9 \%$ & $T T$ & $29.7 \%$ \\
\hline & & $(27 / 70)$ & & $(21 / 69)$ & & $(16 / 67)$ & & $(19 / 64)$ \\
\hline \multirow[t]{4}{*}{ The $4^{\text {th }}$ base } & TC & $32.9 \%$ & GA & $31.7 \%$ & CA & $28.0 \%$ & AA & $26.8 \%$ \\
\hline & & $(24 / 73)$ & & $(26 / 82)$ & & $(21 / 75)$ & & $(19 / 71)$ \\
\hline & TG & $23.3 \%$ & GG & $25.8 \%$ & CC & $33.3 \%$ & $T T$ & $32.4 \%$ \\
\hline & & $(14 / 60)$ & & $(16 / 62)$ & & $(22 / 66)$ & & $(22 / 68)$ \\
\hline
\end{tabular}

in mismatch site (the $2^{\text {nd }}, 3^{\text {rd }}$ and $4^{\text {th }}$ site closest to the SNP site), there exist three different mismatch styles for every kind of base. The best mismatch style is chosen according to the statistical results from Table 4. For example, if there is a $\mathrm{G}$ in the $3^{\text {rd }}$ site, the mismatches of $\mathrm{CC}, \mathrm{CA}$ and $\mathrm{CT}$ will be formed. The polymorphism percent of primers with CA (46.8\%) mismatch is highest compared to that of CC (23.9\%) and CT (32.1\%).
We further identified the usability of the primer design method on rapeseed and sesame. During rapeseed oil content research, with $\mathrm{DH}$ lines of $\mathrm{F} 1$ generation between zy036 and 51070, which had been reported by Hua et al [30], we identified a QTL related to oil content located on A2 chromosome. Because of lack of markers in the QTL interval, we designed a total of 20 SNP primers between marker O110C05 and marker BrSF000036-9 using our

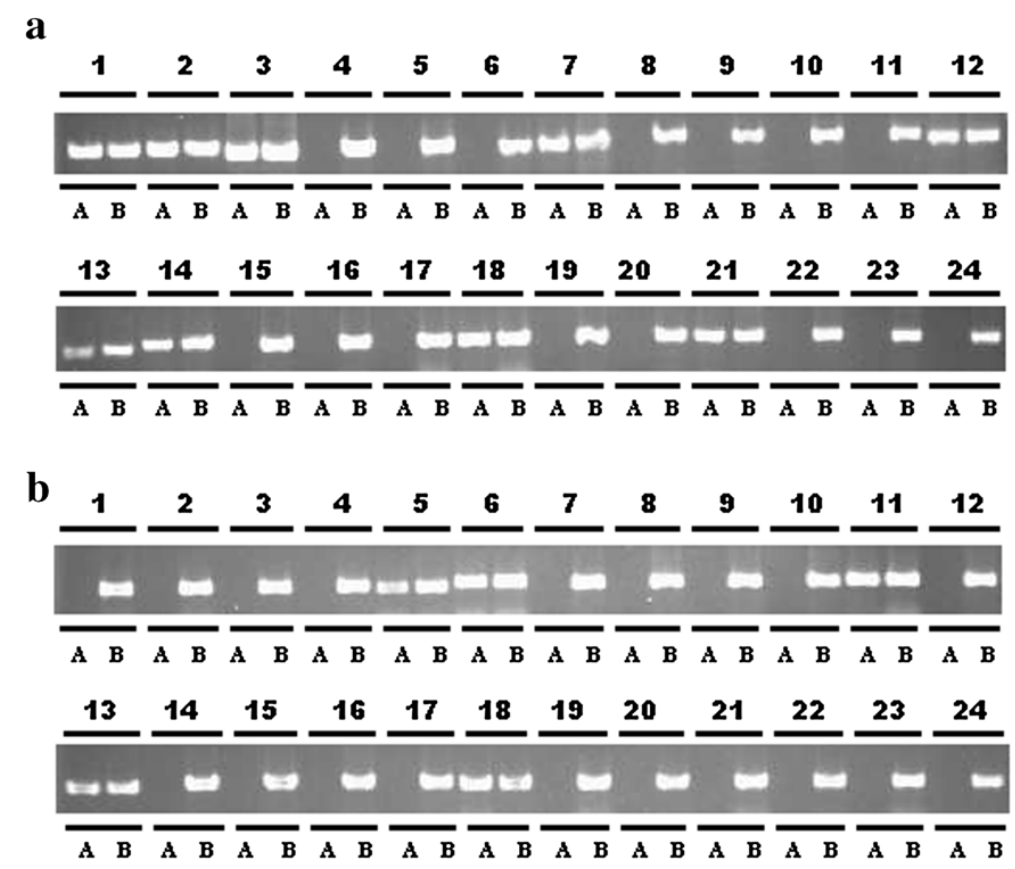

Figure 2 Analysis of specificity for SNP primers of $\boldsymbol{B}$. oleracea. $\mathbf{a}$, 1-24 primer pairs, which corresponded to Bo001-Bo024, were introduced with a CA base pair mismatch in the $3^{\text {rd }}$ nucleotide closest to $3^{\prime}$ end (A/T SNP type, AA mismatch). $\mathbf{b}, 24$ primer pairs, which corresponded to Bo194-Bo217, were introduced with a CA base pair mismatch in the $3^{\text {rd }}$ nucleotide closest to $3^{\prime}$ end (A/T SNP types, TT mismatch). A, 01-88; B, 02-12. 
method (Table 5). Results showed that 15 primers were located in the QTL interval (Figure 3). The polymorphism percent of primers is $(15 / 20,75 \%)$. Another 24 primers came from A/T SNPs between two sesame lines 28-31 and ZZM2289 were also designed. And among them, 18 SNP markers were detected to have polymorphism (18/24, 75\%) (Additional file 3). Both results from rapeseed and sesame indicated that the SNP primer method could be used in other species.

\section{Discussion}

In recent years, various methods for high-throughput SNP analysis have been described [28]. Although these methods are highly efficient compared to other traditional SNP genotyping by electrophoresis, significant investments of expensive probes, microchips or special instrumentation have limited their use in most laboratories. For traditional low-throughput SNP genotyping methods, the main time and labor, and low efficiency of specific primer are still challenges [27]. The Allele-specific PCR method was developed for allele analysis of clinically significant mutations. To facilitate reliable discrimination between two alleles highly, the addition of artificial mismatches within the three bases from 3'end of the primers might be beneficial [23]. Although the third position from the 3'end has been detected as the best to place a mismatch base in primer previously [24], we really do not know which kind of mismatch (for every base, there are three kinds of mismatches) is the best choice in the $3^{\text {rd }}$ position. In this study, different from the previous reports which used only a limited quantity of primers, a large amount of SNP primers designed by introducing mismatches within the three bases closest to the 3'end of primers were used to solve this problem.

Generally, AS-PCR primers designed randomly had a low allelic specificity rate of approximately $30 \%$, which was consistent with our results (29.1\%). However, mismatch sites $\left(2^{\text {nd }}, 3^{\text {rd }}\right.$, and $4^{\text {th }}$ site closest to the $3^{\prime}$ end $)$ had different effects on the polymorphic efficiency of primers. In our study, we found primer polymorphic percent was lowest in the $2^{\text {nd }}$ base location because many primers could not amplify any bands in both of the lines 01-88 and 02-12. For the $4^{\text {th }}$ base location, the polymorphism efficiencies of all mismatch types were almost equivalent (under 30\%). The highest polymorphic percent was found in the $3^{\text {rd }}$ base located closest to the SNP site, which was observed by Hayashi et al similarly (2004) [24].

According to the results of thermodynamics of mismatches reported by Peyret et al (1999) and Little (2001) $[28,29]$, the mismatch base pairs had different destabilization effects that could be divided into weak, medium, and strong strength of destabilization. Therefore, during design of AS-

Table 520 primers pairs designed according to SNPs between rapeseed zy036 and 51070

\begin{tabular}{|c|c|c|c|c|c|}
\hline $\begin{array}{l}\text { Primer } \\
\text { name }\end{array}$ & $\begin{array}{l}\text { SNP } \\
\text { type }\end{array}$ & $\begin{array}{l}\text { Mismatch site } \\
\text { closest to } \\
\text { 3'end of } \\
\text { primers }\end{array}$ & $\begin{array}{c}\text { Mismatch } \\
\text { base pairs }\end{array}$ & Forward primer( $\left(5^{\prime}-3^{\prime}\right)$ & Reverse primer( $\left(5^{\prime}-3^{\prime}\right)$ \\
\hline Br94494 & $\mathrm{C} / \mathrm{G}$ & $2^{\text {nd }}$ & CT & AGTTACATAGGTCCACAATCATAGAATAAACTTTC & TACCATTCGGAGCCTAAATAGAGGTAAAAGGTG \\
\hline Br27005 & T/A & $3^{\text {rd }}$ & CA & AAGATTGTTTCAAACGCAAAAATATACAACAAAAT & CAACGAATTTCACACTTTAGTAATGCACTGAGATTT \\
\hline $\mathrm{Br} 31054$ & T/A & $3^{\text {rd }}$ & CA & CAAGTGAGACTGAATCCACAATAAAGGATGCTACT & TCGGATAAAATCCCCAGCTCTACTATACATTCC \\
\hline $\mathrm{Br} 43193$ & $\mathrm{~T} / \mathrm{A}$ & $3^{\text {rd }}$ & CA & CCTTITATTTGATCACAGGGGTTTGTAGGAACT & TCAGCCAGTAACGTCCCCCACATC \\
\hline Br51190 & $\mathrm{T} / \mathrm{A}$ & $3^{\text {rd }}$ & CA & AAATAATGGCATGCTCCTCTITAATCTACCAAACT & TATTCGGTTCCGAAAATAATGCGATGC \\
\hline Br34590 & $\mathrm{T} / \mathrm{A}$ & $3^{\text {rd }}$ & CA & AAGTGACGGTTCTITAAGTTATCAGAGTCTCCTAAT & AGATTTGGGATTAAAATCAAGTTGTGGGTTAGTTIT \\
\hline Br03637 & $\mathrm{T} / \mathrm{A}$ & $3^{\text {rd }}$ & CA & ATTACAGAATGTGTGTGCAAACAGAAATACATTACT & TGTGTCCCCATTTCGTGTAATCATAAAGCTAG \\
\hline Br39807 & T/A & $3^{\text {rd }}$ & CA & CGAGACTCGGGTCGTTGAGTGGAAAT & ATCCTAAAGACTTCTCCCACAAATCCACCAT \\
\hline Br68275 & T/A & $3^{\text {rd }}$ & CA & TGCCGCATGTATGTCGGAGATGATAAT & AACCGAAACCCTAGTAGGCTAGGCGC \\
\hline Br29253 & T/A & $3^{\text {rd }}$ & CA & TGGCGCTAAATCCAAGAAGAAGTCCATT & AATTACCACCTTTCTTACCCTTGTTACTCATGACAG \\
\hline Br61715 & T/A & $3^{\text {rd }}$ & CA & TGAATAGATTCTTCCGCATCACCTTTTAAAGTTAAT & CTTGTTTCAAGAGAAATTGAACAAGCTGCAGT \\
\hline Br24494 & $C / G$ & $2^{\text {nd }}$ & GT & GTCAATAATACTAGCAAACATACAACAGCGAGATTC & TTGCAAATTTTAGTCAAAGTCGGTAGAAAATAGATC \\
\hline Br06710 & $\mathrm{T} / \mathrm{A}$ & $3^{\text {rd }}$ & CA & TCTTGTCGATGCTGAGCTGGCAAATACT & GGTCAAGCTCACACACACTCCACGTC \\
\hline Br00855 & $\mathrm{T} / \mathrm{A}$ & $3^{\text {rd }}$ & CA & CACTATGGGCTATGGTGGGTCCTTCAAT & TGATTGGAGTTCTGTGCTCGTAGTTTTGC \\
\hline Br71197 & $\mathrm{C} / \mathrm{G}$ & $2^{\text {nd }}$ & GT & TATGGCACACAGACAGAGTTCCAGGAAATC & TCTTCCAGTTCGATATCTTGGTCTGTCCC \\
\hline Br07809 & $\mathrm{C} / \mathrm{G}$ & $2^{\text {nd }}$ & GT & CTCCGCCCACATGTTATAATATGTCAGTATATCTTC & TAGTGAATGGAGAAAGAGAACAAAGCCTACAGTACA \\
\hline Br02456 & T/A & $3^{\text {rd }}$ & CA & GCCTTCAGAAGGTCTGGAAACTGGATT & GTTCGATGGACTTCACTACCTCCCATAGCT \\
\hline Br77080 & T/A & $3^{\text {rd }}$ & CA & CGGATAGTTTCGGGTTCGGTTCGATT & ACCGAACGGGTACCCGAATATATAAAAATATTAATT \\
\hline $\mathrm{Br} 77646$ & T/A & $3^{\text {rd }}$ & CA & TCCACCAGAATTGTGTGATGGCACTTACT & GAAAAACGTCAGGTCAATGTATCAACTTCGATAA \\
\hline $\mathrm{Br} 14184$ & G/A & $3^{\text {rd }}$ & $A C$ & GATTAACCGATGAAAGTCTCAGTGCCACAG & TCGTCTGTGACTCCCAAACACTTGGATAG \\
\hline
\end{tabular}




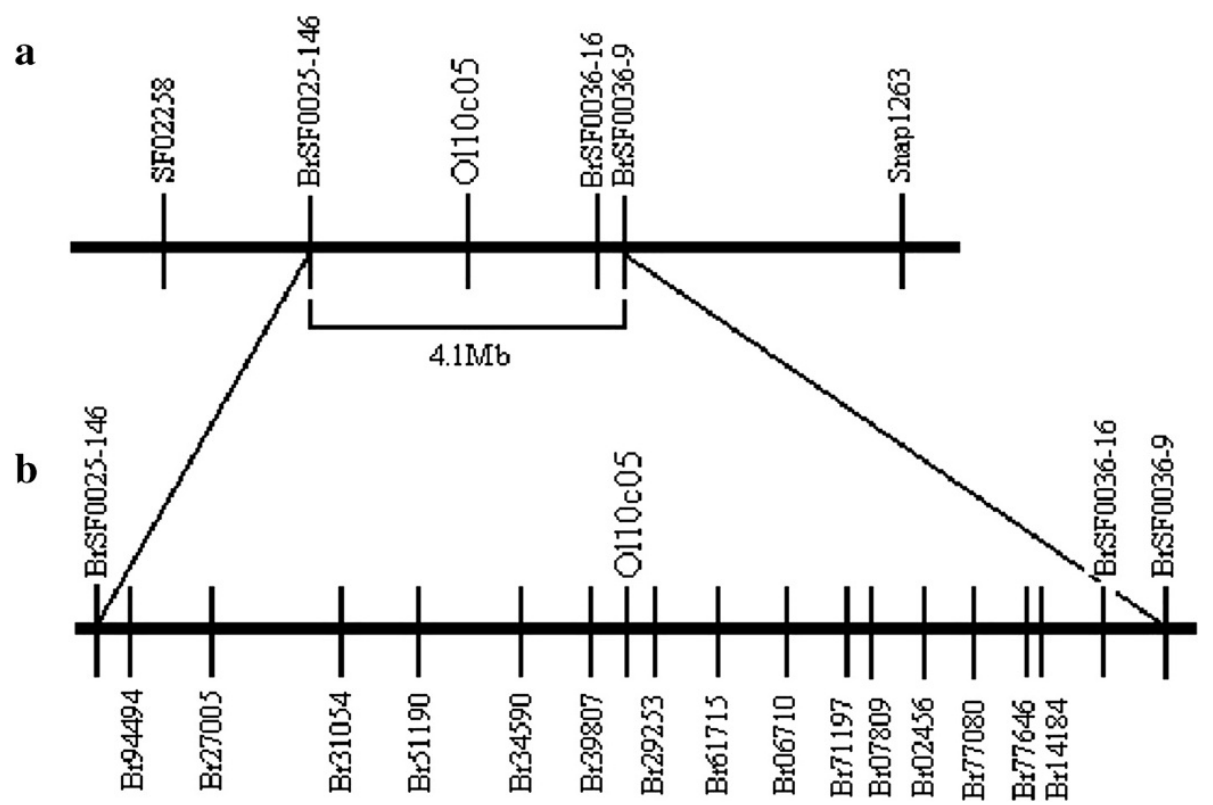

Figure 3 SNP marker density increase of rapeseed linkage map. a, An oil content QTL was scanned between O110C05 and BrSF000036-9. b, Among twenty primers, fifteen new makers were generated for further QTL location.

PCR primers, the effects of mismatches no matter in 3'end or within the three bases closest to the 3'end of primers should be both considered [26,27]. In this study, for convenient analysis, we compressed four SNP types including $\mathrm{A} / \mathrm{T}$ (T/A), A/G (T/C), A/C (T/G), and G/C (C/G) based on their destabilization effects of mismatch base pairs formed in 3'end of primers. Among them, primers generated from SNP types A/G (T/C) had the lowest detection efficiency in all mismatch sites. It was reasonable because AC and GT mismatches had weak destabilization strength. The primers including these specific mismatches at 3'end were easier to make amplification in both alleles.

Similarly, mismatch types within the three bases closest to the 3'end affect specificities of primers. In the $3^{\text {rd }}$ base, CA and TG (the highest polymorphic mismatches) belonged to weak destabilization strength mismatches. The mismatches GA, TC, TT, and CC (the higher polymorphic mismatches) located at the $4^{\text {th }}$ base away from the SNP site belonged to the strong destabilization strength mismatches. From the results, we deduced that SNPs (A/T), which contained CA mismatches in the $3^{\text {rd }}$ nucleotide from the 3 ' end of the primers, had the highest allele-specification. According to the combination rules, polymorphic efficiency between TT (mismatch in 3'end of primer, strong destabilization strength) and CA (weak destabilization strength) are typically higher than AA (mismatch in 3'end of primer, medium destabilization strength) and CA. Our results confirmed this deduction.

Based on these results, we performed the primer design principle which could form the one and only best primer for every SNP type. Among them, mismatches in the second positions were more appropriate for SNP type (C/G and $G / C$ ), which was different from the viewpoint that mismatch in the $3^{\text {rd }}$ position was the best choice for ASPCR. With the primer design principle, we further tested the primers designed based on SNPs of rapeseed and sesame. High efficient polymorphism of the primers identified the usability of the method in other species.

\section{Conclusion}

A SNP primer design method was developed which improved the polymorphism efficiency of AS-PCR primers highly. The modified primer design can help to identify the best effective primer for each SNP and potentially is a valuable tool for gene mapping, map-based cloning and marker-assisted selection in crops.

\section{Methods}

Plant materials and SNP information

At least 20ug genome DNA of B. oleracea lines 01-88 and $02-12$ at a concentration of $\geq 50 \mathrm{ng} / \mathrm{ul}$, was sent for Solexa sequencing as a commercial service. The DNA was fragmented into small pieces using divalent cations at elevated temperature. The cleaved short DNA fragments were prepared for Solexa sequencing in BGI (China). REPEAT MASTER was used for screening repeated sequences with default parameter and labeling the sequences from different materials. For genome location of fragments, SOAP adapting the default parameter values was used for the initial alignment and screening 
to avoid the effects of paralog [31]. SNP primer design was performed using screened results.

\section{SNP analysis and verification by Sanger sequencing}

To verify the putative SNPs, 96 SNP sites derived from only 8-read sequences were randomly chosen between $B$. oleracea lines 01-88 and 02-12. Primers (Sangon, China) were designed to amplify about $500 \mathrm{bp}$ fragments in which containing the corresponding SNPs. The PCR reaction contained $25 \mathrm{ng}$ DNA, $0.2 \mathrm{mM}$ dNTP, 0.5U Taq (MBI, USA) with 1xbuffer, and $5 \mathrm{pM}$ of each primer. PCR parameters were as follows: a pre-denaturation of $94^{\circ} \mathrm{C}$ for $2 \mathrm{~min}$, 35 cycles of amplification $\left(94^{\circ} \mathrm{C}\right.$ for $30 \mathrm{~S}, 60^{\circ} \mathrm{C}$ for $1 \mathrm{~min}$ and $72^{\circ} \mathrm{C}$ for $1 \mathrm{~min}$ ) and a final extension reaction was performed at $72^{\circ} \mathrm{C}$ for $5 \mathrm{~min}$. PCR products were detected on $1.0 \%$ agarose gel by electrophoresis and ligated into PMD18T-vector (Takara, Japan) for SNP identification.

\section{Primer design and testing}

Allele-specific primers corresponding to 12 kinds of SNP in B. oleracea were designed according to different combinations between mismatch base and mismatch site. Optimization of melting temperature, primer length and amplified products length were achieved using primer program WebSNAPER (http://pga.mgh.harvard.edu/cgibin/snap3/websnaper3.cgi) [23]. Primer sequences were screened against $B$. oleracea genome repetitive sequences to minimize mis-priming.

Polymorphism assay of SNP primers were performed by PCR and detected by agarose gel electrophoresis. All the forward primers are allele-specific for $B$. oleracea line $02-12$ and the reverse primer is not allele-specific. Amplification of SNP primers was performed on C1000TM Thermal Cycler (Bio-Rad, USA) using $20 \mathrm{ul}$ reactions. Before carrying out this study, we had chosen some Taq polymerases: MBI Taq DNA Polymerase and Takara Taq (two general Taq), MBI Dream Taq ${ }^{\mathrm{TM}}$ DNA Polymerase and Takara Ex Taq (which are better in amplification efficiency and sensitivity compared to general Taqs) to identify their effects on amplification efficiency. Result showed both of general polymerases (MBI Taq DNA Polymerase and Takara Taq) had same and high allele-specific amplification efficiency compared to the other two Taqs. Therefore, general Taq polymerase would be best choice in allele-specific PCR and Taq DNA Polymerase from MBI was chose in this study. The PCR reaction contained $25 \mathrm{ng}$ DNA, $0.2 \mathrm{mM}$ dNTP, $0.5 \mathrm{U}$ Taq DNA Polymerase with 1 xbuffer, and $5 \mathrm{pM}$ of each primer. PCR parameters were as follows: a predenaturation of $94^{\circ} \mathrm{C}$ for $2 \mathrm{~min}, 35$ cycles of amplification $\left(94^{\circ} \mathrm{C}\right.$ for $30 \mathrm{~s}, 55^{\circ} \mathrm{C}-65^{\circ} \mathrm{C}$ for $1 \mathrm{~min}$ and $72^{\circ} \mathrm{C}$ for $30 \mathrm{~s}$ ) and a final extension reaction was performed at $72^{\circ} \mathrm{C}$ for $10 \mathrm{~min}$. PCR products were separated on $2.5 \%$ agarose gel by electrophoresis.

\section{Application in rapeseed and sesame}

Rapeseed DNA samples including two parents (high oil content line zy036 and low oil content line 51070) and DH lines, which had been reported by Hua et al [30], were prepared using the DNAeasy plant kit miniprep (Qiagen, Valencia, CA). Zy036 and 51070 were resequenced and blasted with $B$. napus genome sequence (unpublished). Additionally, two sesame lines 28-31 and ZZM2289 (genome sequence has not been published) were also used in our research. All SNPs were chosen according to the method described in B. oleracea lines 01-88 and 02-12. The SNP primers were designed according to our primer design method.

\section{Additional files}

Additional file 1: 1686 primer pairs designed based on the SNPs between $B$. oleracea lines 01-88 and 02-12.

Additional file 2: 385 primer pairs designed based on the SNPs between $B$. oleracea lines 01-88 and 02-12 which are A/T SNP type with $\mathrm{G}$ and $\mathrm{T}$ base in the $3^{\text {rd }}$ nucleotide.

Additional file 3: 24 primer pairs designed based on the SNPs between sesame lines 28-31 and ZZM2289.

\section{Abbreviations}

SNP: Single Nucleotide Polymorphism; AS-PCR: Allele-Specific PCR; SSR: Simple Sequence Repeat; RFLP: Restriction fragment length polymorphism; AFLP: Amplified fragment length polymorphism; CAPs: The Cleaved Amplified Polymorphic Sequence; dCAPs: Derived The Cleaved Amplified Polymorphic Sequence.

\section{Competing interests}

The authors declare that they have no competing interests.

\section{Authors' contributions}

$J \mathrm{~L}$ and SMH contributed to primer design and took the co-lead role in writing the manuscript. SMH and MYS performed the primer analysis. SYL offered sequence data of B. oleracea and B. napus. YML and WWX provided the DNA of B. oleracea 01-88 and 02-12. XRZ provided the genome sequence and DNA of S. indicum L. 28-31 and ZZM2289. HZW participated in discussions during experimental work. WH conceived the project and approved the final version of the manuscript. All authors read and approved the final manuscript.

\section{Acknowledgement}

This study was supported by the National Key Basic Research Program of China (2011CB109300), National 863 plans projects (2012AA101107), and Key Projects in the National Science \& Technology Pillar Program (2010BAD01B02)

\section{Author details}

${ }^{1}$ Key Laboratory of Biology and Genetic Improvement of Oil Crops, Ministry of Agriculture, Oil Crops Research Institute of the Chinese Academy of Agricultural Sciences, Wuhan 430062, People's Republic of China. ${ }^{2}$ Institute of Vegetables and Flowers of the Chinese Academy of Agricultural Sciences, Beijing 100081, People's Republic of China.

Received: 15 June 2012 Accepted: 17 August 2012

Published: 24 August 2012

\section{References}

1. Gut IG: Automation in genotyping of single nucleotide polymorphisms. Hum Mutat 2001, 17:475-492.

2. Kwok PY: Methods for genotyping single nucleotide polymorphisms. Annu Rev Genomics Hum Genet 2001, 2:235-258. 
3. Flint-Garcia SA, Thornsberry JM, Buckler ES: Structure of linkage disequilibrium in plants. Annu Rev Plant Biol 2003, 54:357-374.

4. Wang C, Liu Z: Arabidopsis ribonucleotidereductases are critical for cell cycle progression, DNA damage repair, and plant development. Plant Cell 2006, 18:350-365.

5. Feltus FA, Wan J, Schulze SR, Estill JC, Jiang N, Paterson AH: An SNP resource for rice genetics and breeding based on subspecies indica and japonica genome alignments. Genome Res 2004, 14:1812-1819.

6. Hillier LW, Miller RD, Baird SE, Chinwalla A, Fulton LA, Koboldt DC, Waterston $\mathrm{RH}$ : Comparison of $\mathrm{C}$. elegans and $\mathrm{C}$. briggsae genome sequences reveals extensive conservation of chromosome organization and synteny. PLOS Biol 2007, 5:e167.

7. Eberle MA, Ng PC, Kuhn K, Zhou L, Peiffer DA, Galver L, Viaud-Martinez KA, Lawley CT, Gunderson KL, Shen R, Murray SS: Power to detect risk alleles using genome-wide tag SNP panels. PLOS Genet 2007, 3:1827-1837.

8. Shendure J, Mitra RD, Varma C, Church GM: Advanced sequencing technologies: methods and goals. Nat Rev Genet 2004, 5:335-344.

9. Barbazuk WB, Emrich SJ, Chen HD, Li L, Schnable PS: SNP discovery via 454 transcriptome sequencing. Plant J 2007, 51:910-918.

10. Trick M, Long Y, Meng J, Bancroft I: Single nucleotide polymorphism (SNP) discovery in the polyploid Brassica napus using Solexa transcriptome sequencing. Plant Biotechnol J 2009, 7:334-346.

11. Li R, Li Y, Fang X, Yang H, Wang J, Kristiansen K, Wang J: SNP detection for massively parallel whole-genome resequencing. Genome Res 2009, 19:1124-1132.

12. Livak KJ, Marmaro J, Todd JA: Towards fully automated genome-wide polymorphism screening. Nat Genet 1995, 9:341-342.

13. Myakishev MV, Khripin Y, Hu S, Hamer DH: High-throughput SNP genotyping by allele-specific PCR with universal energy-transfer-labeled primers. Genome Res 2001, 11:163-169.

14. Huang J, Wei W, Zhang J, Liu G, Bignell GR, Stratton MR, Futreal PA, Wooster $\mathrm{R}$, Jones KW, Shapero MH: Whole genome DNA copy number changes identified by high density oligonucleotide arrays. Hum Genomics 2004, 1:287-299.

15. Shapero $\mathrm{MH}$, Zhang J, Loraine A, Liu W, Di X, Liu G, Jones KW: MARA: a novel approach for highly multiplexed locus-specific SNP genotyping using high-density DNA oligonucleotide arrays. Nucleic Acids Res 2004, 32:e181.

16. Matsuzaki H, Dong S, Loi H, Di X, Liu G, Hubbell E, Law J, Berntsen T, Chadha M, Hui H, Yang G, Kennedy GC, Webster TA, Cawley S, Walsh PS, Jones KW, Fodor SP, Mei R: Genotyping over 100,000 SNPs on a pair of oligonucleotide arrays. Nat Methods 2004, 1:109-111.

17. Shen R, Fan JB, Campbell D, Chang W, Chen J, Doucet D, Yeakley J, Bibikova M, Wickham Garcia E, McBride C, Steemers F, Garcia F, Kermani BG, Gunderson K, Oliphant A: High-throughput SNP genotyping on universal bead arrays. Mutat Res 2005, 573:70-82

18. Konieczny A, Ausubel FM: A procedure for mapping Arabidopsis mutations using co-dominant ecotype-specific PCR-based markers. Plant J 1993, 4:403-410.

19. Neff MM, Turk E, Kalishman M: Web-based primer design for single nucleotide polymorphism analysis. Trends Genet 2002, 18:613-615.

20. Thiel T, Kota R, Grosse I, Stein N, Graner A: SNP2CAPS: a SNP and INDEL analysis tool for CAPS marker development. Nucleic Acids Res 2004, 32:e5.

21. Cha RS, Zarbl H, Keohavong P, Thilly WG: Mismatch amplification mutation assay (MAMA): application to the C-H-ras gene. PCR Methods App/ 1992, 2:14-20.

22. Kwok S, Chang SY, Sninsky JJ, Wang A: A guide to the design and use of mismatched and degenerate primers. PCR Methods Appl 1994, 3:S39-S47.

23. Drenkard E, Richter BG, Rozen S, Stutius LM, Angell NA, Mindrinos M, Cho RJ, Oefner PJ, Davis RW, Ausubel FM: A simple procedure for the analysis of single nucleotide polymorphisms facilitates map-based cloning in Arabidopsis. Plant Physiol 2000, 124:1483-1492.

24. Hayashi K, Hashimoto N, Daigen M, Ashikawa I: Development of PCR-based SNP markers for rice blast resistance genes at the Piz locus. Theor Appl Genet 2004, 108:1212-1220.

25. Hirotsu N, Murakami N, Kashiwagi T, Ujiie K, Ishimaru K: Protocol: a simple gel-free method for SNP genotyping using allele-specific primers in rice and other plant species. Plant Methods 2010, 6:12.

26. Wangkumhang P, Chaichoompu K, Ngamphiw C, Ruangrit U, Chanprasert J, Assawamakin A, Tongsima S: WASP: a Web-based Allele-Specific PCR assay designing tool for detecting SNPs and mutations. BMC Genomics 2007, 8:275

27. Bui M, Liu Z: Simple allele-discriminating PCR for cost-effective and rapid genotyping and mapping. Plant Methods 2009, 5:1.

28. Tsuchihashi Z, Dracopoli NC: Progress in high throughput SNP genotyping methods. Pharmacogenomics J 2002, 2:103-110.

29. Little S: Amplification-refractory mutation system (ARMS) analysis of point mutations. Curr Protoc Hum Genet 2001, 9:9.8.1-9.8.12.

30. Hua W, Li RJ, Zhan GM, Liu J, Li J, Wang XF, Liu GH, Wang HZ: Maternal control of seed oil content in Brassica napus: the role of silique wall photosynthesis. Plant J 2012, 69:432-444.

31. Li R, Li Y, Kristiansen K, Wang J: SOAP: short oligonucleotide alignment program. Bioinformatics 2008, 24:713-714

doi:10.1186/1746-4811-8-34

Cite this article as: Liu et al.: An improved allele-specific PCR primer design method for SNP marker analysis and its application. Plant Methods 2012 8:34

\section{Submit your next manuscript to BioMed Central and take full advantage of:}

- Convenient online submission

- Thorough peer review

- No space constraints or color figure charges

- Immediate publication on acceptance

- Inclusion in PubMed, CAS, Scopus and Google Scholar

- Research which is freely available for redistribution 